\title{
Características de una población sobreexplotada de concha navaja, Ensis macha, en Bahía Independencia, Perú, durante el 2004
}

\author{
Overfishing population characteristics of razor clam, Ensis macha, from \\ Independencia Bay, Peru, in 2004 year
}

\author{
Roberto Espinoza ${ }^{1}$, Juan Tarazona ${ }^{1}$ y Jürgen Laudien ${ }^{2}$
}

\begin{abstract}
1Laboratorio de Ecología Marina, Facultad de Ciencias Biológicas, Universidad Ciencias Biologicas, Universidad nacional Mayor de San Marcos, Apartado 110058 - Lima 11. Email Juan Tarazona: jltarazona@yahoo.com.

Email Roberto Espinoza: respinoza. unmsm@gmail.com

2 División de Biociencias, Alfred Wegener Institut für Polar und
\end{abstract} Meeresforschung (AWI)

Presentado: $\quad$ 02/03/2010 Aceptado: $\quad$ 21/10/2010 Publicado online: 21/01/2011

\begin{abstract}
Resumen
Muestreos cuantitativos mensuales, durante el año 2004, fueron realizados para estimar la densidad y biomasa de una población de Ensis macha (Molina 1782) en Morro Quemado, Bahía Independencia. Se estudian las relaciones biométricas, los parámetros de crecimiento y de producción poblacional, utilizando la rutina ELEFAN I y el método de Crisp. Durante el 2004 se encontró valores significativamente mayores de la tasa de explotación $\left(E=0,69 \mathrm{a}^{-1}\right)$ y una duplicación de los desembarques mensuales (máximo de $335 \mathrm{t}$ ) y esfuerzo pesquero (máximo de 848 viajes). Estos cambios repercutieron en el mismo año con una disminución significativa de la densidad promedio de la población $\left(\mathrm{D}=54,13\right.$ ind. $\mathrm{m}^{-2}$ ) y la reducción a la mitad de los valores de producción somática $\left(\mathrm{P}=81,99 \mathrm{gPSLC} \mathrm{m}^{-2} \mathrm{a}^{-1}\right)$ y tasa P/B $\left(0,38 \mathrm{a}^{-1}\right)$, respecto a los valores del año anterior. También se observó un incremento significativo de la tasa de mortalidad total $\left(2,84 \mathrm{a}^{-1}\right)$ y una duplicación de la tasa de mortalidad por pesca $\left(1,97 \mathrm{a}^{-1}\right)$, respecto al año anterior. Se concluye que la población estudiada de $E$. macha era un recurso comercial sobreexplotado en el 2003 y que el incremento de la presión pesquera en el 2004, particularmente durante el segundo semestre, intensificó su condición de recurso sobreexplotado.
\end{abstract}

Palabras clave: Concha navaja, Pesca artesanal, Sobrepesca, Motobombas hidráulicas, producción.

\section{Abstract}

Quantitative monthly samplings, in 2004, were carried out to estimate the density and biomass of Ensis macha (Molina 1782), from Morro Quemado area, Bay Independence, Pisco. The present study analyzes biometric relationships, growth parameters and somatic production using the ELEFAN I routine and the Crisp's method. During the year 2004 the rate of exploitation $\left(E=0.69 \mathrm{y}^{-1}\right)$ was significantly bigger than the values of 2003 and the monthly landings (maximum of $335 \mathrm{t}$ ) and fishing effort (maximum of 848 trips) were twice increased. These changes induced in the same year a significant decrease of population mean density $\left(D=54.13\right.$ ind. $\left.\mathrm{m}^{-2}\right)$ and the halfway reduction of somatic production $\left(P=81.99 \mathrm{gAFDW} \mathrm{m}^{-2} \mathrm{y}^{-1}\right)$ and $\mathrm{P} / \mathrm{B}$ rate $\left(0.38 \mathrm{y}^{-1}\right)$ values, regarding the values of the previous year. Also, a significant increment of total mortality rate $\left(2.84 \mathrm{y}^{-1}\right)$ and the fishing mortality rate $\left(1.97 \mathrm{y}^{-1}\right)$ were twice increased, regarding the previous year. It is assumed that the population of razor clams already was a overfishing commercial resource in the 2003 and that the increment of fishing pressure in the 2004, particularly during the second semester, intensified its overfishing resource condition.

Keywords: Razor clam, artisanal fisheries, overfishing, clam kicking, production.

\section{Introducción}

En el Perú la concha navaja, Ensis macha (Molina 1782), comenzó a ser registrada en la pesquería artesanal desde el año 2000, en el área de Pisco, principalmente en la zona de Morro

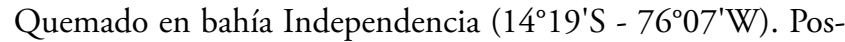
teriormente fue registrada desde San Juan de Marcona (15 $22^{\prime} \mathrm{S}$ - 7509'W) hasta bahía Samanco (09 $\left.10^{\circ} \mathrm{S}-78^{\circ} 33^{\prime} \mathrm{W}\right)$, y en el 2006 además en bahía Sechura (05\%49'S - 8101'W); siendo considerada en la actualidad como un importante recurso bentónico. Desde el año 2003 es reportado el uso de motobombas hidráulicas en la extracción de $E$. macha, arte que incrementa la extracción hasta en cuatro veces con respecto a la forma tradicional (J. Zavala, comunicación personal). En el año 2005 el Ministerio de la Producción de Perú prohibió el uso de las motobombas (R.M. Nº25-2005-PRODUCE, 2 de febrero de 2005) y fueron establecidas vedas con la finalidad de recuperar el recurso en las diferentes áreas de extracción y en particular la población de Morro Quemado (R.M. No266-2005-PRODUCE, 6 de octubre de 2005).

Los estudios sobre la biología o ecología de $E$. macha son escasos en Perú (Espinoza 2006), sin embargo se puede encontrar información sobre el crecimiento, producción e índices reproductivos de poblaciones en Chile y Argentina (Urban 1996, Aracena et al. 2003; Barón et al. 2004), la cual nos permitirá evaluar el estado de las poblaciones peruanas, que en la actualidad pueden ser consideradas en colapso.

En el presente trabajo son evaluadas las características de la población E. macha del área de Morro Quemado (bahía Independencia, Pisco, Perú), durante el año 2004; año en la que ocurrió la mayor intensidad de extracción pesquera sobre la concha navaja.

\section{Material y métodos}

Obtención y procesamiento de muestras.- La población de E. macha fue muestreada mensualmente de enero a diciembre del 2004, en la zona de Morro Quemado, en bahía Independencia (14¹9'35”S, 7607'43”W) (Fig. 1).

Se realizaron dos tipos de muestreos, uno cuantitativo usando un marco cuadrado de $0,5 \mathrm{~m}$ de lado, con 5 réplicas en cada una de las estaciones de muestreo, ubicadas a profundidades de 4; 8 y $12 \mathrm{~m}$. Otro muestreo cualitativo, también mensual, consistió en la colecta ad libitum de 40 - 80 ejemplares.

En cada individuo fue determinada la longitud $(\mathrm{L}=$ distancia del borde anterior al borde posterior, con un vernier $\pm 0,05 \mathrm{~mm}$ ), el peso total (PT), peso húmedo de visceras (PHV) y peso seco de visceras (PSV) con una balanza de $\pm 0,01 \mathrm{~g}$ de precisión. El peso seco se obtuvo colocando los tejidos blandos en una estufa 


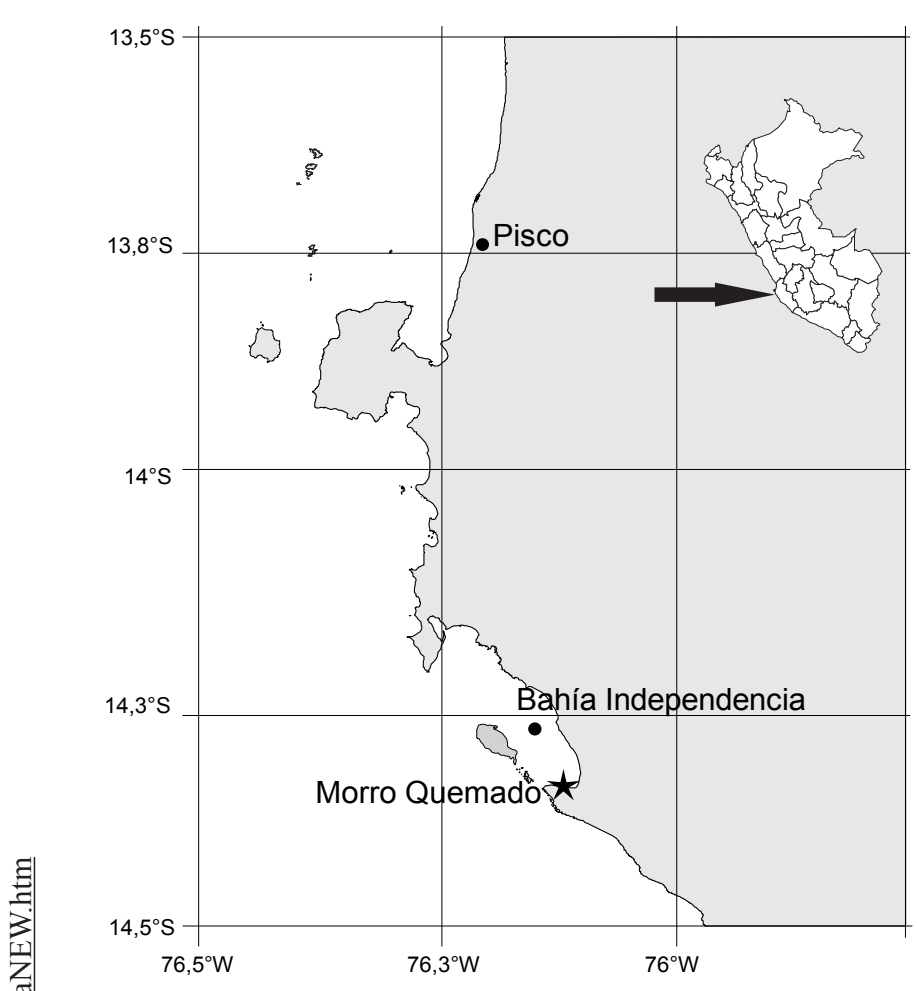

Figura 1. Ubicación del área de muestreo de la concha navaja, Ensis macha, en Morro Quemado, Bahía Independencia durante el año 2004

$\left(90^{\circ} \mathrm{C}\right.$; $48 \mathrm{~h}$ ). El peso seco libre de cenizas (PSLC) fue obtenido incinerando las partes blandas y secas de los individuos en una mufla $\left(600^{\circ} \mathrm{C} ; 3 \mathrm{~h}\right)$ y pesadas en una balanza analítica $( \pm 0,0001$ g). Adicionalmente, se tomaron datos de temperatura, oxígeno y clorofila a, usando un equipo perfilador CTD.

\section{Análisis de datos y estadística}

Pesquería.- Para evaluar la actividad pesquera fueron utilizadas las estadísticas de desembarques y esfuerzos de pesca del año 2004 para el área de estudio (expresado en número de viajes y buzos dedicados a la extracción de la concha navaja), proporcionados por el IMARPE (Yamashiro com. pers.).

Estructura poblacional.- Las relaciones longitud-peso total, peso húmedo visceral, peso seco visceral y peso seco libre de cenizas se determinaron utilizando la ecuación de alometría Ps $=a L t^{b}$, la cual fue ajustada por el método de los mínimos cuadrados. Se determinó el peso estándar mensual y promedio (g PT) para un individuo de longitud de $150 \mathrm{~mm}$, que puede ser considerada la talla comercial (Aracena et al. 1998).

Se analizó la distribución mensual de tallas agrupadas en intervalos de $5 \mathrm{~mm}$, y se hallaron las variables estadísticas de longitud máxima observada, longitud promedio, moda, varianza y desviación estándar.

\section{Dinámica poblacional}

Crecimiento y mortalidad. -Para la estimación de los parámetros de crecimiento fueron utilizados los datos mensuales de frecuencia de tallas del 2004, mediante el software FISAT (Gayanilo et al. 1995). ELEFAN I (Pauly \& David 1981) permitió estimar los parámetros de crecimiento $\mathrm{K}_{ } \mathrm{L}_{\infty}$ a partir de la frecuencia de tallas, en base a la identificación de grupos de edad en cada muestra, obteniéndose una curva de crecimiento dada por la función de crecimiento no oscilante de von Bertalanffy:

$\mathrm{L}_{\mathrm{t}}=\mathrm{L}_{\infty}\left(1-\mathrm{e}^{-\mathrm{K}\left(\mathrm{t}-\mathrm{t}_{0}\right)}\right)$,

donde:

$\mathrm{L}_{\mathrm{t}}$ es la longitud de la valva (talla) a la edad $t$,

$\mathrm{L}_{\infty}$ es la media asintótica de la longitud de la valva,

$\mathrm{K}$ es la constante de crecimiento en ańos ${ }^{-1}$,

t es la edad en años, y

$\mathrm{t}_{0}$ es la edad a la longitud cero.

Para la determinación del valor de $\mathrm{t}_{0}$, que representa el tiempo en que el organismo tiene cero milimetros de longitud, se utilizó la siguiente ecuación de acuerdo a Pauly (1983):

$\log \left(-\mathrm{t}_{0}\right)=-0,3922-0,2752^{*} \log \left(\mathrm{L}_{\infty}\right)-1,038^{*} \log (\mathrm{K})$

Como método de comparación de los parámetros estimados, en base a la relación estadística entre $\mathrm{K}_{ } \mathrm{L}_{\infty}$, con otros reportados en la literatura, fue estimado un índice de rendimiento del crecimiento $\Phi$ (Pauly \& Munro 1984) usando la ecuación:

$$
\Phi=\log (\mathrm{K})+2^{*} \log \left(\mathrm{L}_{\infty}\right)
$$

La mortalidad total Z (año ${ }^{-1}$ ) fue estimada a partir de los datos de frecuencia de tallas, mediante el método de curva de captura basada en la conversión de tallas (Pauly 1983). Usando los parámetros de crecimiento del modelo de von Bertalanffy, las longitudes de todas las navajas agrupadas en intervalos de tallas fueron convertidas a edad mediante la ecuación:

$$
\mathrm{N}_{\mathrm{j}} / \Delta \mathrm{t}_{\mathrm{j}}=\mathrm{N}_{0} \mathrm{e}^{-\mathrm{Ztj}}
$$

donde:

$\mathrm{N}_{\mathrm{j}}$ es el número de individuos en el intervalo de talla $\mathrm{j}$,

$\Delta \mathrm{t}_{\mathrm{j}}$ es el tiempo necesario para crecer a través del intervalo $\mathrm{j}$,

$\mathrm{N}_{0}$ es el número de individuos en el tiempo 0 .

$t_{j}$ es la edad relativa correspondiente a la talla media del intervalo de $\mathrm{j}$.

La edad $t_{j}$ fue calculada utilizando el modelo de crecimiento de von Bertalanffy inverso:

$$
\begin{aligned}
& \mathrm{t}_{\mathrm{j}}=(1 / \mathrm{K}) \operatorname{Ln}\left(1-\mathrm{L}_{\mathrm{j}} / \mathrm{L}_{\infty}\right)+\mathrm{t}_{0}, \\
& \text { donde: }
\end{aligned}
$$

Hj es la altura de la valva (talla) a la edad $t_{j}$.

La mortalidad $\mathrm{Z}$ fue finalmente calculada mediante un análisis de regresión lineal de la ecuación:

$$
\begin{aligned}
& \operatorname{Ln}\left(N_{j} / \Delta t_{j}\right)=\operatorname{Ln}\left(N_{0}\right)-Z_{j} \\
& \text { donde: }-b=Z
\end{aligned}
$$

La mortalidad natural $\mathrm{M}\left(\mathrm{anno}^{-1}\right)$ fue estimada de acuerdo a Hewitt y Hoenig (2005), mediante la ecuación:

$$
\begin{aligned}
& \mathrm{M}=4,22 / \mathrm{E}_{\text {max }} \\
& \text { donde } \mathrm{E}_{\max } \text { es la edad máxima. }
\end{aligned}
$$

La mortalidad por pesca $\mathrm{F}\left(\right.$ año $\left.^{-1}\right)$ y la tasa de explotación $\mathrm{E}$ $\left(\mathrm{año}^{-1}\right)$, fueron calculadas mediante las ecuaciones:

$$
\mathrm{F}=\mathrm{Z}-\mathrm{M} \text { y } \mathrm{E}=\mathrm{F} / \mathrm{Z}
$$


Tabla 1. Comparación de los parámetros de la ecuación Longitud $=a \mathrm{Peso}^{\mathrm{b}}$ para la concha navaja Ensis macha en Bahía Independencia durante el año 2004 con valores de la literatura. Peso total (PT), Peso húmedo visceral (PHV) y Peso seco visceral (PSV).

\begin{tabular}{ccccccc}
\hline $\mathbf{a}$ & $\mathbf{b}$ & $\mathbf{r}^{2}$ & $\mathbf{N}$ & Relación & Localidad & Referencia \\
\hline 2,00E-06 & 3,34 & 0,75 & 554 & Longitud-PT & Bahía Independencia, Perú & Espinoza 2006 \\
2,00E-07 & 3,73 & 0,73 & 474 & Longitud-PHV & Bahía Independencia, Perú & Espinoza 2006 \\
3,00E-10 & 4,68 & 0,69 & 475 & Longitud-PSV & Bahía Independencia, Perú & Espinoza 2006 \\
1,00E-05 & 3,07 & 0,73 & 810 & Longitud-PT & Bahía Independencia, Perú & Este estudio \\
3,00E-05 & 2,75 & 0,58 & 389 & Longitud-PHV & Bahía Independencia, Perú & Este estudio \\
6,00E-06 & 2,73 & 0,48 & 386 & Longitud-PSV & Bahía Independencia, Perú & Este estudio \\
2,90E-07 & 2,93 & 0,74 & 718 & Longitud-PT & Chile Central & Aracena et al. 1998 \\
8,84E-03 & 3,22 & 0,99 & 243 & Longitud-PT & Chile Central & Lépez et al. 1997 \\
5,32E-03 & 3,35 & 0,98 & 221 & Longitud-PT & Chile Central & Aracena et al. 1998 \\
1,00E-05 & 3,12 & 0,98 & 662 & Longitud-PT & Tubul-Chile & Universidad Austral de Chile 1998. \\
1,00E-05 & 3,10 & 0,98 & 226 & Longitud-PT & Corral-Chile & Universidad Austral de Chile 1998. \\
1,04E-05 & 3,03 & 0,86 & 270 & Longitud-PT & Golfo San Matías, Argentina & Barón et al. 2004 \\
3,79E-05 & 2,66 & 0,68 & 270 & Longitud-PHV & Golfo San Matías, Argentina & Barón et al. 2004 \\
\hline
\end{tabular}

Donde:

Z: mortalidad total

M: mortalidad natural

Producción secundaria.- La producción somática de la población $\mathrm{P}\left(\mathrm{en} \mathrm{g}\right.$ PSLC $\mathrm{m}^{-2}$ año $\left.^{-1}\right)$ fue calculada por el método de la tasa de crecimiento específico en peso (Crisp 1984) usando la distribución de frecuencia de tallas, los parámetros VBGF y las relaciones longitud-PSLC:

$$
P=\Sigma N_{i} W_{i} G_{i}
$$

donde: $\mathrm{N}_{\mathrm{i}}=$ número promedio de ejemplares ( $\mathrm{N}^{\circ} \mathrm{Ind} \cdot \mathrm{m}^{-2}$ )

$\mathrm{W}_{\mathrm{i}}=$ peso corporal promedio (en g, PSLC) en la clase de longitud i

$\mathrm{G}_{\mathrm{i}}=$ tasa de crecimiento específico en peso $\left(\right.$ año $\left.{ }^{-1}\right)$ :

$\mathrm{G}_{\mathrm{i}}=\mathrm{b} \mathrm{K}\left(\left(\mathrm{L}_{\infty} / \mathrm{L}_{\mathrm{i}}\right)-1\right)$

donde $b$ es el exponente de la relación longitud-peso, $\mathrm{L}_{\infty} \mathrm{y}$ $\mathrm{K}$ son los parámetros VBGF y $\mathrm{L}_{\mathrm{i}}$ es la longitud promedio en la clase de longitud i.

La biomasa promedio anual $\overline{\mathrm{B}}$ (en g PSLC por $\mathrm{m}^{2}$ por año) fue hallada por:

$$
\overline{\mathrm{B}}=\Sigma \mathrm{N}_{\mathrm{i}} \mathrm{W}_{\mathrm{i}}
$$

La relación anual $\mathrm{P} / \mathrm{B}$ fue calculada a partir de la producción somática anual $\mathrm{P}$ y de la biomasa media anual B.

\section{Resultados}

Condiciones oceanográficas.- Ensis macha habita en los fondos arenosos del submareal de Morro Quemado y se distribuye desde 3 a $18 \mathrm{~m}$ de profundidad. El 2004 la temperatura de fondo tuvo un promedio de $15,1^{\circ} \mathrm{C}$ (enero-marzo) y de $14,3{ }^{\circ} \mathrm{C}$ (julio-setiembre) durante el verano e invierno, respectivamente. Las concentraciones de oxígeno disuelto cerca al fondo fueron más bajas en el verano, fluctuando entre 0,5 y $1,0 \mathrm{~mL} . \mathrm{L}^{-1}$, en relación al resto del año $\left(1,0-3,5 \mathrm{~mL} \cdot \mathrm{L}^{-1}\right)$. Los valores de clorofila- $a$ fueron altos durante todo el período de estudio, con valores mayores de $10 \mu \mathrm{g} \cdot \mathrm{L}^{-1}$.

\section{Estructura poblacional}

Pesquería, densidad y biomasa.- En el año 2004 se observó una tendencia descendente de la densidad y biomasa poblacional. La densidad disminuyó desde valores máximos en enero $(77,87$ ind. $\left.\mathrm{m}^{-2}\right)$, hasta valores mínimos en diciembre $\left(22,93\right.$ ind. $\left.\mathrm{m}^{-2}\right)$, con promedio de 54,13 ind. $\mathrm{m}^{-2}$. La biomasa fluctuó entre 2,95 $\mathrm{kg} \cdot \mathrm{m}^{-2}$ (enero 2004$)$ y $1,35 \mathrm{~kg} \cdot \mathrm{m}^{-2}$ (diciembre 2004), con un promedio de 2,51 kg.m $\mathrm{m}^{-2}$ (Fig. 2).

Los desembarques registraron una tendencia ascendente desde enero $(98,18 \mathrm{t})$ a diciembre $(335,02 \mathrm{t})$. De igual forma, se observó en el esfuerzo pesquero, con valores de 287 (enero

Tabla 2. Parámetros de crecimiento estimados para la navaja Ensis macha en Bahía Independencia durante el año 2004 y comparados con

\begin{tabular}{|c|c|c|c|c|c|c|c|}
\hline Especie & $\mathrm{L}_{\infty}(\mathrm{mm})$ & $\mathbf{K}$ & $\mathbf{t}_{0}$ & $\Phi$ & Localidad & Método & Referencia \\
\hline E. macha & 184,10 & 0,48 & $-0,39$ & 4,21 & Bahía Independencia, Perú & AFT & Espinoza 2006 \\
\hline E. macha & 184,70 & 0,55 & $-0,33$ & 4,27 & Bahía Independencia, Perú & AFT & Este estudio \\
\hline E. macha & 196,73 & 0,30 & $\mathrm{n}, \mathrm{d}$, & 4,07 & Chile Central & AFT & Aracena et al. 1998 \\
\hline E. macha & 189,90 & 0,21 & $-0,58$ & 3,88 & Chile Central & $\mathrm{AFT}$ & Urban 1996 \\
\hline E. macha & 216,51 & 0,25 & $-0,28$ & 4,07 & Chile Central & AFT & Aracena et al. 1998 \\
\hline E. macha & 154,00 & 0,25 & $-0,08$ & 3,77 & Golfo San Matías, Argentina & OAC & Barón et al. 2004 \\
\hline E. macha & 153,70 & 0,20 & $-0,72$ & 3,67 & Golfo San Matías, Argentina & OAC & Barón et al. 2004 \\
\hline E. arcuatus & 139,80 & 0,37 & n.d. & 3,86 & Bahía de Orphir, Irlanda & $\mathrm{OAC}$ & Robinson y Richardson 1998 \\
\hline E. arcuatus & 166,60 & 0,26 & n.d. & 3,85 & Bahía de Irlanda, Irlanda & OAC & Robinson y Richardson 1998 \\
\hline E. ensis & 131,60 & 0,57 & n.d. & 3,99 & Gales del Norte, Inglaterra & OAC & Robinson y Richardson 1998 \\
\hline E. siliqua & 154,70 & 0,54 & n.d. & 4,11 & Gales del Norte, Inglaterra & OAC & Robinson y Richardson 1998 \\
\hline E. siliqua & 139.60 & 0.65 & n.d. & 4,10 & Barrinha. Portugal & OAC & Gaspar et al. 1994 \\
\hline
\end{tabular}
valores obtenidos con otras especies del Ensis. Métodos: análisis de frecuencia de tallas (AFT) y observación de anillos de crecimiento (OAC). 

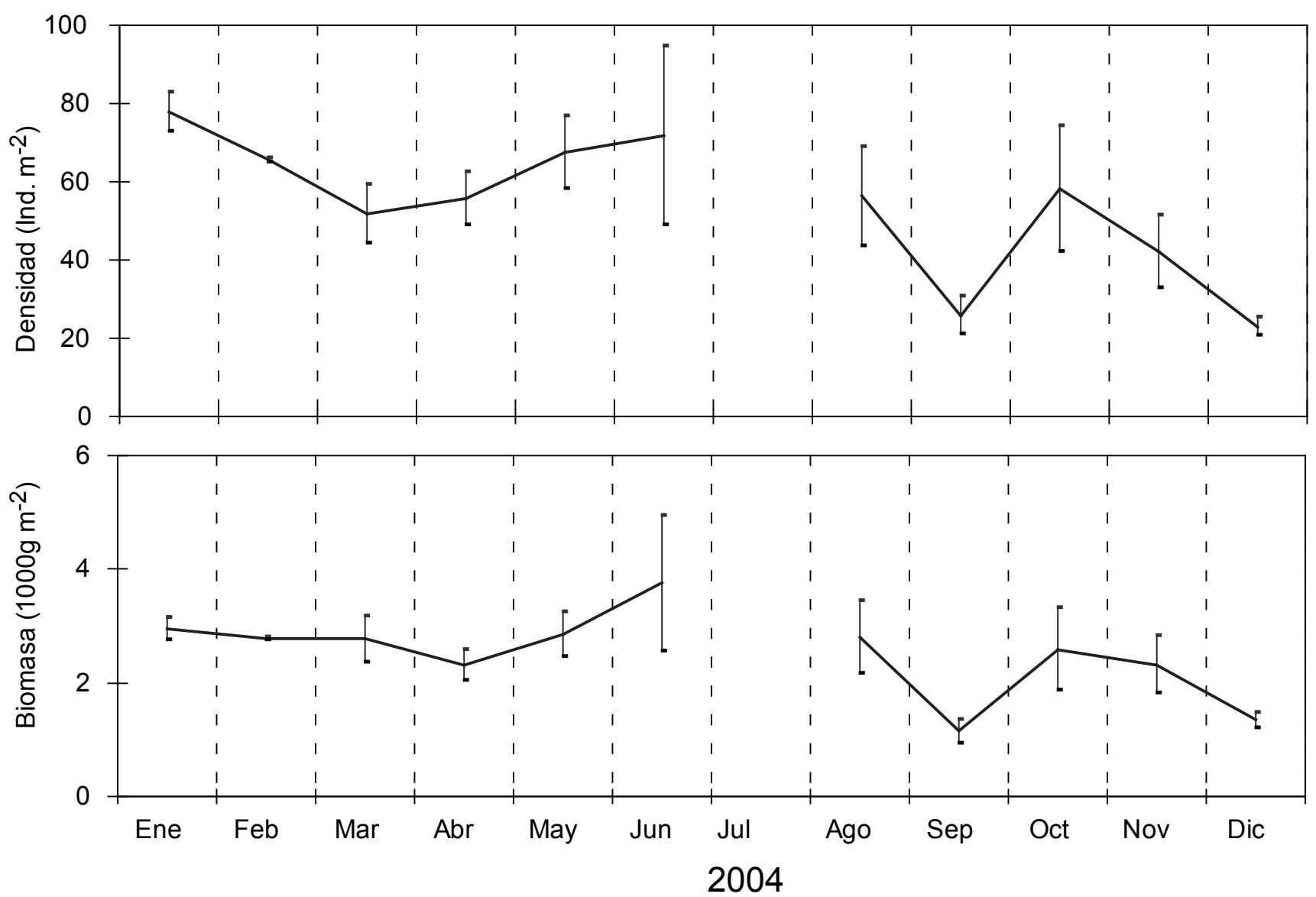

Figura 2. Densidad y biomasa promedio mensual de la concha navaja Ensis macha en Morro Quemado, Bahía Independencia durante el año 2004

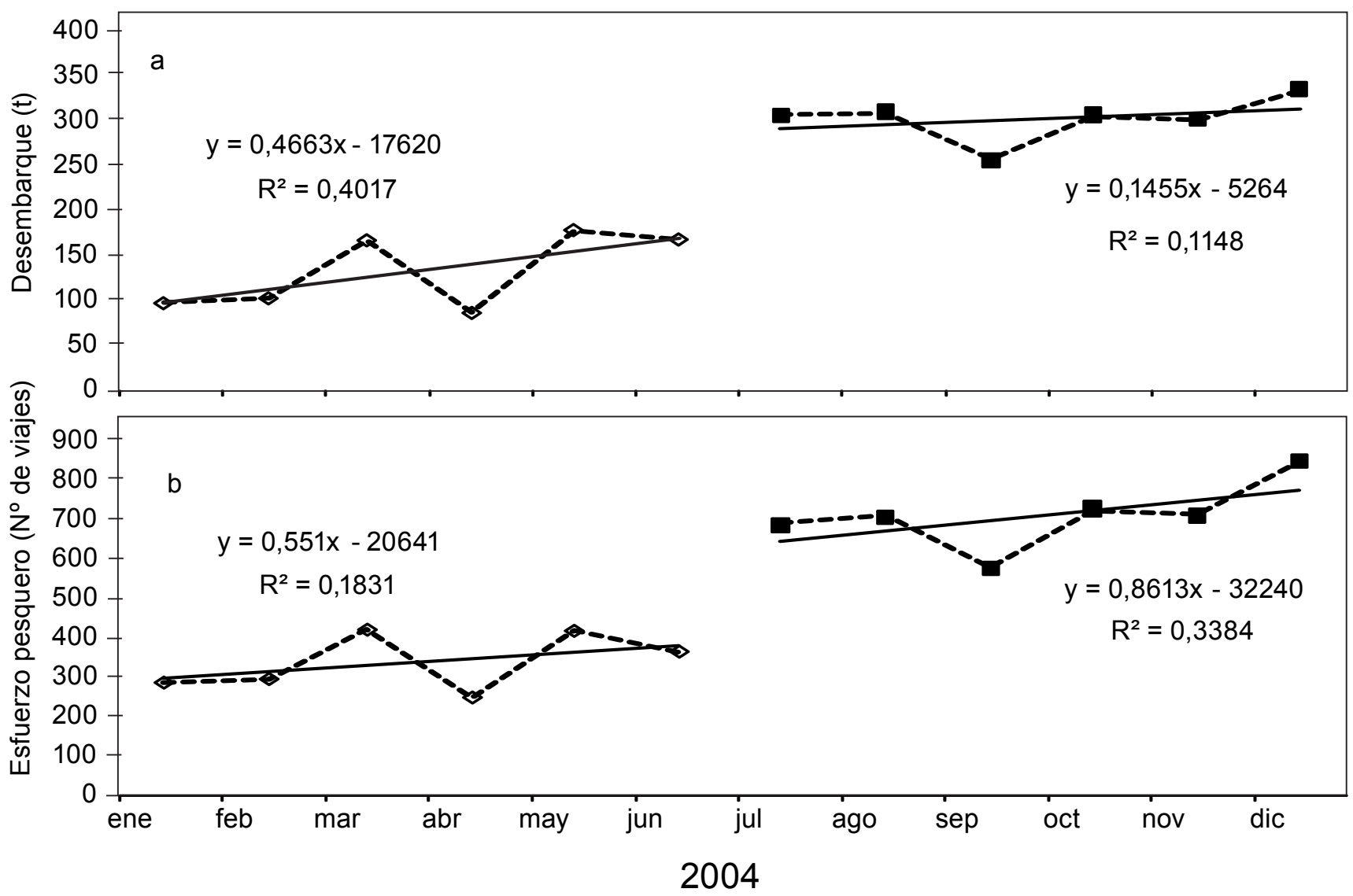

Figura 3. Desembarque (a) y esfuerzo pesquero (b) de la concha navaja Ensis macha en Morro Quemado, Bahía Independencia, durante el año 2004. 


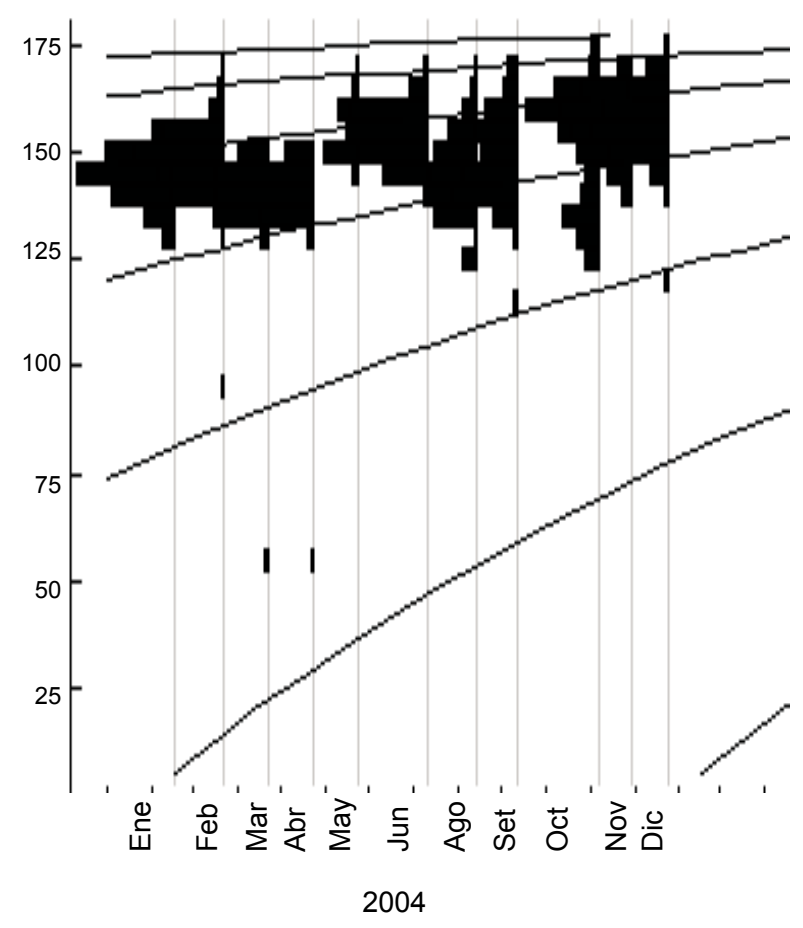

Figure 4. Curva de crecimiento de la navaja Ensis macha en base a frecuencia de tallas dada por la rutina FISAT, durante el año 2004.

2004) a 848 viajes (diciembre 2004) y de 894 (enero) a 4901 buzos (diciembre) dedicados a la extracción de la concha navaja (Fig. 3a, b).

La actividad pesquera muestra dos etapas bien diferenciadas durante el año 2004; el primer semestre (enero-junio 2004) caracterizado por desembarques menores a $180 \mathrm{t}$ y un esfuerzo menor a 425 viajes (Fig. 3a) y el segundo trimestre (juliodiciembre 2004), con desembarques relativamente altos entre 260 a 335 y una mayor esfuerzo pesquero con valores entre 580 y 850 viajes (Fig. 3b).

Se observó una correlación significativa inversa entre desembarque y densidad $(\mathrm{p}=0,029)$, pero no con la biomasa. De la misma forma, se evidenció una correlación significativa inversa entre el esfuerzo pesquero (número de viajes y número de buzos) y la densidad mensual para el año 2004 ( $\mathrm{p}<0,05)$.
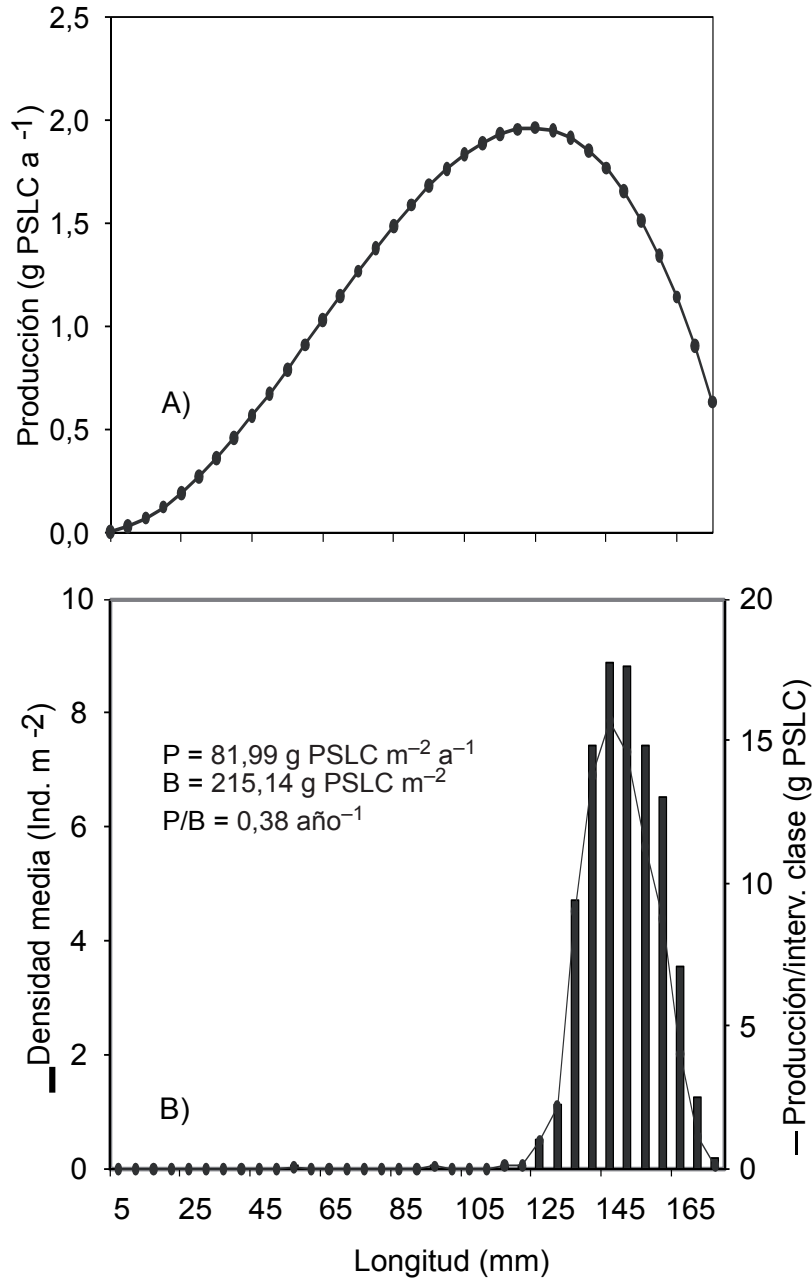

Figure 5. Producción somática individual y poblacional, y tasa de renovación poblacional $(\mathrm{P} / \mathrm{B})$ de la navaja Ensis macha de Morro Quemado, Bahía Independencia, durante el año 2004.

Estructura de tallas y relación talla-peso.- La estructura de tallas de la población tuvo un rango entre 90 y 175 mm para el período de estudio, con notoria ausencia de la fracción juvenil. La distribución de tallas fue unimodal $(150 \mathrm{~mm})$. La longitud máxima y promedio observada fue de 172 y 146,5 mm $\pm 10,8$ (EE), respectivamente.

Tabla 3. Parámetros de la producción secundaria obtenidos de la navaja Ensis macha en Bahía Independencia y comparados con otros valores de bivalvos del Pacífico Sudeste.

\begin{tabular}{lcccccc}
\hline Especie & $\mathbf{B}\left(\mathbf{g}\right.$ PSLC $\left.\mathbf{~}^{-2}\right)$ & Psom $\left(\mathbf{g}\right.$ PSLC $\left.\mathbf{~ m}^{-2} \mathbf{~} \mathbf{r}^{-1}\right)$ & $\mathbf{P} / \mathbf{B}\left(\mathbf{a}^{-1}\right)$ & Período & Localidad & Referencia \\
\hline Gari solida & 2,69 & 27,60 & 0,33 & $1991-92$ & Chile & Urban \& Campos 1994 \\
G. solida & 189,30 & 107,90 & 0,57 & 1990 & Perú & Urban \& Tarazona 1996 \\
G. solida & 71,60 & 21,30 & 0,30 & 1992 & Perú & Urban \& Tarazona 1996 \\
G. solida & 23,50 & 14,00 & 0,60 & 1993 & Perú & Urban \& Tarazona 1996 \\
G. solida & 14,40 & 8,60 & 0,60 & 1994 & Perú & Urban \& Tarazona 1996 \\
Protothaca thaca & 2,30 & 16,90 & 0,27 & $1991-92$ & Chile & Urban \& Campos 1994 \\
Semele solida & 1,91 & 4,80 & 0,19 & $1991-93$ & Chile & Urban \& Campos 1994 \\
Tagelus dombeii & 26,70 & 7,80 & 0,29 & $1991-94$ & Chile & Urban 1996 \\
Venus antiqua & 69,56 & 40,69 & 0,59 & 1994 & Chile & Clasing et al. 1994 \\
V. antiqua & 122,00 & 22,00 & 0,18 & $1991-92$ & Chile & Urban 1996 \\
E. macha & 43,60 & 9,70 & 0,22 & $1991-92$ & Chile & Urban 1996 \\
E. macha & 275,92 & 191,07 & 0,69 & 2003 & Perú & Espinoza 2006 \\
E. macha & 215,14 & 81,99 & 0,38 & 2004 & Perú & Presente estudio \\
\hline
\end{tabular}


La relación talla-peso fue mejor descrita por una regresión potencial para todos los pesos usando PT, PHV, PSV Y PSLC (Tabla 1). Los pesos promedio de individuos fluctuaron entre $37,89 \mathrm{~g}$ (enero 2004) a 58,80 g (diciembre 2004). Se observó una correlación significativa directa entre el peso individual y el desembarque y esfuerzo pesquero $(\mathrm{p}<0,05)$.

El peso estándar promedio calculado para una talla comercial de $150 \mathrm{~mm}$ en el 2004 fue de 47,91 g, mientras los pesos estándares mensuales fluctuaron entre $41,60 \mathrm{~g}$ (noviembre 2004) a 67,08 g (marzo 2004).

\section{Dinámica poblacional}

Crecimiento y mortalidad.- Se obtuvo una tasa de crecimiento $(\mathrm{K})$ de $0,55 \mathrm{año}^{-1}$, una longitud infinita $\left(\mathrm{L}_{\infty}\right)$ de 184,7 mm y un $t_{0}=-0,33\left(R_{n}=0,242\right)$ para el período 2004 (Fig. 4; Tabla 2). La curva teórica de crecimiento mostró que la mayor parte de la población estudiada $(135-150 \mathrm{~mm})$ tuvo una edad entre 2 y 2,75 años para este estudio. La edad máxima y masa máxima fueron calculados en 4,87 años y 6,47 g PSLC, respectivamente, correspondiente a una longitud máxima observada de $172 \mathrm{~mm}$. El índice de rendimiento del crecimiento fue estimado en $\Phi=4,27 \mathrm{a}^{-1}$.

La tasa de mortalidad total $(Z)$ estimada por la curva de captura fue de $2,84 \mathrm{a}^{-1}$. De acuerdo al modelo de Hewitt y Hoenig (2005), la tasa de mortalidad natural fue de $0,87 \mathrm{a}^{-1}$. La mortalidad por pesca $(\mathrm{F})$ y la tasa de explotación (E) fueron estimadas en 1,97 y $0,69 \mathrm{a}^{-1}$, respectivamente.

Producción secundaria.- Los estimados de producción fueron calculados a partir de una biomasa anual por clase de 215,14 g PSLC $\mathrm{m}^{-2}$ y la regresión longitud-PSLC $\left[\mathrm{y}=1 \mathrm{e}-06 \mathrm{x}^{3,05}\left(\mathrm{r}^{2}=\right.\right.$ $0,50 ; n=400)$ ]. La producción individual de la concha navaja en el 2004 alcanzó $1,97 \mathrm{~g}$ PSLC a ${ }^{-1}$ a los $125 \mathrm{~mm}$, decreciendo luego paulatinamente en las tallas mayores.

Las navajas comprendidas entre 140 y $160 \mathrm{~mm}$ de longitud fueron las más abundantes y por tanto las que aportaron significativamente a la producción somática poblacional de $81,99 \mathrm{~g}$ PSLC $\mathrm{m}^{-2} \mathrm{a}^{-1}$, siendo escasa la contribución de tallas menores a la producción (Fig. 5; Tabla 3). La tasa P/B para el 2004 fue estimada en 0,38 año $^{-1}$.

\section{Discusión}

La pesquería de la concha navaja Ensis macha constituye una actividad artesanal importante debido a su consumo y exportación. Sin embargo, en Bahía Independencia esta pesquería disminuyó drásticamente y casi colapsó como consecuencia de la sobreexplotación y falta de regulación pesquera (Mendo \& Espinoza 2008).

La densidad promedio de E. macha en Morro Quemado, observada en los años 2002 y 2003 fueron de 150,98 y de 79,7 ind. $\mathrm{m}^{-2}$, respectivamente; posiblemente causadas por las condiciones oceanográficas favorables, propias del sistema peruano de surgencias costeras, y al estado incipiente de esta pesquería (Espinoza 2006). Durante el año 2004 la densidad promedio decreció hasta 50,6 ind. $\mathrm{m}^{-2}$, principalmente relacionado con el incremento de las capturas y del esfuerzo pesquero.

Aunque la biomasa no mostró una correlación significativa con las variables pesqueras, si disminuyó respecto a los años anteriores, de $2,9 \mathrm{~kg} \cdot \mathrm{m}^{-2}$ en el 2003 a $2,5 \mathrm{~kg} \cdot \mathrm{m}^{-2}$ en el 2004
(Espinoza 2006). La escasa correlación de la biomasa con las variables pesqueras podría ser explicada por el mayor peso individual estimado en el 2004 y el incremento de biomasa en la etapa de maduración gonádica.

La estructura por tallas de E. macha durante el 2004 en bahía Independencia fue similar al reportado en el 2003, con un rango comprendido entre 90 - 175 mm (Espinoza, 2006), lo que indica que se trataba de una población compuesta sólo de individuos adultos.

La máxima longitud de $E$. macha observada en el estudio $\left(\mathrm{L}_{\max }\right.$ ${ }_{2004}=172 \mathrm{~mm}$ ) también fue menor que las reportadas en poblaciones de Chile Central (185 - 220 mm; Universidad Austral de Chile 1998). Esta diferencia podría explicarse por la presión pesquera y la falta de un plan de ordenamiento en Perú, en contraste con la pesquería chilena que si contaba con regulación pesquera; pero también, Urban \& Tesch (1996) han postulado una posible gradiente latitudinal, con individuos más grandes en latitudes más altas; hipótesis que queda por comprobar.

Por otro lado, la ausencia de tallas pequeñas durante el estudio puede explicarse por muchos factores, como la falta de un desove intenso, la alta mortalidad larval, el reducido asentamiento exitoso y fallas en el patrón de dispersión larval y otros efectos debidos al impacto pesquero sobre el recurso.

Consideramos que la hipótesis más factible sería una falla en el patrón de dispersión y asentamiento larval dentro de la bahía, asociados al disturbio del fondo por la intensa actividad extractiva, ya que se han encontrado especímenes juveniles en zonas alejadas de la población estudiada y sin impacto de la pesquería (Espinoza 2006).

La tasa de crecimiento de la navaja peruana de 0,55 en el 2004 resultó similar que lo estimado en el 2003 ( $K=0,48$, Espinoza 2006), pero mayor que las tasas calculadas para poblaciones chilenas (Canales y Ponce 1995, Urban 1996, Urban y Tesch 1996, Aracena et al. 1998) y argentinas (Barón et al. 2004).

Por otro lado, la edad máxima de 4,87 años, encontrada en el presente trabajo, resultó significativamente menor al de las poblaciones chilenas (10 años, Aracena et al. 1998 y Universidad Austral de Chile 1998; 14 años, Urban 1996), demostrando una mayor tasa de crecimiento en la población peruana de concha navaja, lo que podría ser explicado por las condiciones ambientales favorables del ecosistema peruano de surgencias costeras (Tarazona \& Arntz 2001). Estos aspectos concordarian con el hecho de que especies de zonas tropicales o subtropicales tienen una tasa de crecimiento mayor y alcanzan la longitud infinita en un menor tiempo, al contrario de las ubicadas en latitudes más altas (Urban \& Tesch 1996).

Por otro lado, se estimó una tasa de mortalidad total y de pesca de $2,84 \mathrm{a}^{-1}$ y $1,97 \mathrm{a}^{-1}$ en el 2004 que resultaron considerablemente mayores que las observadas en el 2003, con valores de $1,94 \mathrm{a}^{-1}$ y $0,98 \mathrm{a}^{-1}$ (Espinoza 2006). Esto debido a una alta tasa de explotación en el $2004\left(E=0,69 a^{-1}\right)$, que resulta mayor que la estimada en el $2003\left(E=0,60 a^{-1}\right)$. Según Caddy y Csirke (1983), una tasa de explotación de 0,5 es la tasa óptima de explotación de una pesquería, por lo cual es una evidencia más de que la población de E. macha en el 2003 ya estaba sobreexplotada y que en el 2004 se intensificó con el cambio de arte de pesca. 
Las estadísticas de la pesquería de la concha navaja en el 2002 y 2003 demuestran desembarques que variaron entre valores entre 47 y 190 t, y entre 44 y 180 t, respectivamente, que resultaron mayores a los de años anteriores. Mientras que, en el 2004 los valores de desembarque fueron mayores, entre 64 y 335 t, lo cual estuvo también acorde con los más altos valores de esfuerzo pesquero registrados en la bahía. Además, podemos destacar que el número de viajes dedicados a la extracción de la concha navaja en la bahía durante los meses de julio a diciembre del 2004 (entre 582 a 848) fue casi el doble que el del primer semestre (entre 249 a 422). Este importante incremento del esfuerzo pesquero fue debido al cambio de arte de pesca en las embarcaciones pesqueras durante el segundo semestre, cuando se generalizó el uso de un sistema de motobomba o "clam kicking" (Mendo \& Espinoza 2008, Gaspar et al. 2008). Esta mayor eficiencia del arte permitió mayores capturas del recurso, con lo cual aumentó la presión pesquera en el área (Espinoza 2006), y se incrementó la mortalidad por pesca. De esta forma el mayor impacto pesquero en la estructura poblacional, sobre todo a nivel de densidad y biomasa, se dio en el segundo semestre del 2004.

El cambio de arte de pesca sería también el responsable del incremento de la mortalidad natural, ya que la presión de agua, generada por la motobomba, no sólo mataría directamente los individuos al dañar sus valvas, sino que ademas muchos individuos no serían capaces de enterrarse y quedarían vulnerables a los depredadores o morirían por lesiones o stress, lo cual se traduciría en una importante mortalidad post-pesca, (Gaspar et al. 1994, Robinson \& Richardson 1998). Asimismo, Tuck et al. (2000) demostraron el impacto de la presión de agua de las motobombas como un factor físico disturbante sobre el sustrato y la comunidad biológica asociada a Ensis spp.

En cuanto a la producción secundaria, la producción somática poblacional de $E$. macha disminuyó significativamente desde valores de 191,07 $\mathrm{g}$ PSLC m ${ }^{-2} \mathrm{a}^{-1}$ en el 2003 (Espinoza 2006) a $81,99 \mathrm{~g} \mathrm{PSLC} \mathrm{m}^{-2} \mathrm{a}^{-1}$ en el 2004, evidentemente asociado al incremento de la presión pesquera. Si se postula que la producción poblacional es igualmente dependiente de la tasa de crecimiento de su biomasa (Benke 1993), se puede sugerir que la menor producción en el 2004 estuvo relacionada sobre todo a menores valores de densidad y la ausencia de individuos juveniles. Sin embargo, el valor de producción de la navaja del 2004 fue relativamente más alto que el de otros bivalvos dentro de la región del Pacífico Sudeste (Urban \& Tarazona 1996).

Asimismo la tasa P/B durante el año 2003 mostró un valor de $0,69 \mathrm{a}^{-1}$ (Espinoza 2006), pero decayó significativamente a $0.38 \mathrm{a}^{-1}$ en el 2004, debido a las bajas densidades y alta mortalidad por pesca. Sin embargo, estos valores que fueron más altos que los hallado en Chile (0,22 $\mathrm{a}^{-1}$; Urban 1996); y los estimados para otros bivalvos del Pacífico Sudeste, tales como G. solida, Semele solida y Protothaca thaca (Urban \& Campos 1994, Urban \& Tarazona 1996), Venus antiqua (Clasing et al. 1994), V. antiqua y Tagelus dombeii (Urban 1996). Se ha postulado que altos valores de $\mathrm{P} / \mathrm{B}$ están relacionados a altas tasas de mortalidad total, baja masa promedio y altas tasas de crecimiento (Lomovasky et al. 2002).

Se concluye que la población de concha navaja de bahía Independencia ya mostraba indicios de sobreexplotación en el 2003 y el elevado esfuerzo pesquero y desembarque del recurso en el año 2004, sobre todo a partir del segundo semestre en que se cambió el arte de pesca, intensificó su condición de recurso sobreexplotado. Es recomendable mantener el seguimiento de los parámetros biológicos y pesqueros de esta población, para establecer medidas efectivas de regulación pesquera y de recuperación de la población.

\section{Agradecimientos}

Al Instituto Alfred Wegener para Estudios Marinos y Polares (Alemania), a la Universidad Nacional Mayor de San Marcos y al proyecto CENSOR, que brindaron el apoyo académico, financiero y logístico para el desarrollo de esta investigación. A los integrantes del Grupo DePSEA, Laboratorio de Ecología Marina, quienes colaboraron en el trabajo de campo, análisis de datos y sugerencias en la elaboración de esta evaluación.

\section{Literatura citada}

Aracena O., M. Carmona \& L. Medina. 1998. La navaja en la VIII región. Documento No1, Proyecto FONDEF d96/1095. Instituto de Fomento Pesquero, Universidad de Concepción, Chile. 14 pp.

Aracena O., I. Lépez, J. Sánchez, A. Carmona, I. Medina \& A. Saavedra. 2003. On two new macroscopic indexes to evaluate the reproductive cycle of Ensis macha (Molina, 1782). Journal of Shellfish Research 2(3): 675-680.

Avellanal M., E. Jaramillo, E. Clasing, P. Quijón \& H. Contreras. 2002. Reproductive cycles of the bivalves Ensis macha (Molina, 1782) (Solenidae), Tagelus tombeii (Lamarck, 1818) (Solecurtidae), and Mulinia edulis (King, 1831) (Mactridae) in southern Chile. The veliger 45(1): 33-44.

Barón P., L. Real L, N. Ciocco \& M. Ré. 2004. Morphometry, growth and reproduction of an Atlantic population of the razor clam Ensis macha (Molina, 1782). Scientia Marina, 68(2): 211-217.

Benke A.C. 1993. Concepts and patterns of invertebrate production in running waters. Verh Internat. Verein. Limnol. 25: 15-38.

Caddy, J.F. \& J. Csirke. 1983. Approximations to sustainable yield for exploited and unexploited stocks. Océanogr.trop. 18(1):3-15

Clasing E, T. Brey, R. Stead, J. Navarro, G. Ascencios. 1994. Population dynamics of Venus antiqua (Bivalvia: Veneracea) in the Bahía de Yaldad, Isla de Chiloé, southern Chile. J Exp Mar Bio Ecol 177:171-186.

Crisp D. 1984. Energy flow measurements. In: N. Holme and A. McIntyre, eds. Methods for the study of marine benthos. Blackwell, Londres. Pp. 284-372.

Espinoza R. 2006. Estructura y dinámica poblacional de Ensis macha (Molina, 1782) en Bahía Independencia, Pisco, Perú. Tesis para optar el título profesional de Biólogo, Mención en Hidrobiología y Pesquería, Universidad Nacional Mayor de San Marcos, Perú. 61pp.

Gaspar M., C.A. Richardson \& C.C. Monteiro. 1994. The effects dredging on shell formation in the razor clam Ensis siliqua from Barrinha, southern Portugal. J. Mar. Bio. Ass. UK 74: 927-938.

Gaspar M.B., R. Constantino, A. Guerra \& S. Carvalho. 2008. Capítulo 10: Impactos medioambientales de las pesquerías de navajas y longueirones en función de las técnicas de pesca y de los hábitos de explotación. En: A. Guerra y C. Lodeiros. Navajas y longueirones: biología, pesquerías y cultivo. Xunta de Galicia, Consellería de Pesca e Asuntos Marítimos. Pp. 223-269.

Gayanilo F.C., P. Sparre \& D. Pauly. 1995. The FAO-ICLAM Stock Assessment Tools (FISAT) User's Guide. FAO Computerized Information. Series Fisheries 6. 126 pp. 
Guzmán N., S. Saá \& L. Ortlieb. 1998. Catálogo descriptivo de los moluscos litorales (Gastropoda y Pelecypoda) de la zona de Antofagasta, $23^{\circ} \mathrm{S}$ (Chile). Estudios Oceanológicos 17: $17-86$

Hewitt D.A. \& J.M. Hoenig. 2005. Comparison of two approaches for estimating natural mortality based on longevity. Fishery Bulletin US 103: 433-437.

Lépez I., O. Aracena, A. Carmona, et al. 1997. Caracterización bioeconómica de las pesquerías de huepo (Ensis macha) y navajuela (Tagelus dombeii) en la VIII región. Informe Final Proyecto FIP 95-20A. Convenio U. de ConcepciónFEREPA Bio Bio, Chile. Fondo de Investigación Pesquera, Subsecretaría de Pesca. Ministerio de Fomento, Economía y Reconstrucción, Chile. 229 pp. <http://www.fip.cl/ FIP/Archivos/pdf/informes/IT\%2095-20a.pdf $>$ Acceso: 02/03/2010

Lomovasky B., T. Brey, E. Morriconi \& J. Calvo. 2002. Growth and production of the venerid bivalve Eurhomalea exalbida in the Beagle Channel, Tierra del Fuego. Journal of Sea Research 48: 209-216.

Mendo J. \& R. Espinoza. 2008. Capítulo 16: Pesquería y algunos aspectos biológicos de la navaja (Ensis macha) en Perú. En: A. Guerra y C. Lodeiros. Navajas y longueirones: biología, pesquerías y cultivo. Xunta de Galicia, Consellería de Pesca e Asuntos Marítimos. Pp. 359-378.

Paredes C., J. Tarazona, E. Canahuire, L. Romero \& O. Cornejo. 1988. Invertebrados macrobénticos del área de Pisco, Perú. En: H. Salzwedel y A. Landa, eds. Recursos y dinámica del ecosistema de afloramiento peruano. Boletín Instituto del Mar del Perú, Callao, Volumen Extraordinario. Pp. 121-132.

Pauly D. \& J.L. Munro. 1984. Once more on growth comparison in fish and invertebrates. Fishbyte 2: 21.

Pauly D. \& N. David. 1981. ELEFAN I, a basic program for the objective extraction of growth parameters from lengthfrequency data. Meeresforschung 28(4): 205-211.

Pauly D. 1983. Algunos métodos simples para la evaluación de recursos pesqueros tropicales. Fao, Doc. Tec. Pesca 234. 49 pp.
Robinson R.F. \& C.A. Richardson CA. 1998. The direct and indirect effects of suction dredging on a razor clam (Ensis arcuatus) population. Journal of Marine Science 55:970-977

Tarazona, J. \& W. Arntz. 2001. The Peruvian Coastal Upwelling System. En: Ecological Studies. Coastal Marine Ecosystems of Latin America. U. Seeliger and B. Kjerfve (eds). Berlín, p. 229-244.

Tuck I.D., N. Bailey, M. Harding, G. Sangster, T. Howell, N. Graham \& M. Breen. 2000. The impact of water jet dredging for razor clams, Ensis spp., in a shallow sandy subtidal environment. Journal of Sea Research 43 (1): 65-81.

Universidad Austral de Chile. 1998. Estudio biológico pesquero de los recursos almeja, navajuela y huepo en la VIII y X regiones. Proyecto FIP-IT/96-46. Informe Técnico FIP. Fondo de Investigación Pesquera, Subsecretaria de Pesca, Ministerio de Fomento, Economía y Reconstrucción, Chile. $<$ http://www.fip.cl/FIP/Archivos/pdf/informes/IT\%20 96-46.pdf $>$ Acceso:02/03/2010.

Urban H.J. \& B. Campos B. 1994. Population Dynamics of the bivalves Gari solida, Semele solda and Protothaca thaca population from a small bay in Chile at $36^{\circ} \mathrm{S}$. Mar. Ecol. Prog. Ser. 115: 93-102.

Urban H.J. \& C. Tesch. 1996. Aspects of the population dynamics of six bivalve species from Southern Chile. Results of the Victor Hensen Cruise to the Magellan Strait and the Beagle Channel in October/November 1994. Arch. Fish. Mar. Res. 44: 243-256.

Urban H.J. \& J. Tarazona. 1996. Effects of El Niño/Southern Oscillation on the population dynamics of a Gari solida population (Bivalvia: Psammobiidae) from Bahía Independencia, Perú. Mar. Biol. 125:725-734.

Urban H.J. 1996. Population dynamics of the bivalves Venus antiqua, Tagelus dombeii, and Ensis macha from Chile at $36^{\circ} \mathrm{S}$. J. Shellfish Research 15: 719-727. 